\title{
ASPECTOS CINÉTICOS DA DEGRADAÇÃO DE LAMINADOS DE MADEIRAS EM AMBIENTES AQUÁTICO E TERRESTRE
}

\author{
APRILE, F. M., ${ }^{1}$ DELITTI, W. B. C. ${ }^{2}$ e BIANCHINI JR., I. ${ }^{3}$ \\ ${ }^{1} \mathrm{CPRH}$ - Cia. Pernambucana do Meio Ambiente \\ ${ }^{2}$ Departamento de Ecologia Geral, I. B., USP, SP \\ ${ }^{3}$ Departamento de Hidrobiologia, Universidade Federal de São Carlos, São Carlos, SP \\ Correspondência para: Fábio Marques Aprile, Departamento de Hidrobiologia, \\ Universidade Federal de São Carlos, São Carlos, SP \\ Recebido em 21/11/97 - Aceito em 21/10/98 - Distribuído em 10/09/99
}

(Com 5 figuras)

\section{ABSTRACT \\ Kinetics aspects of wood sheets decay on aquatic and terrestrial environments}

Decomposition rates of several wood especies was evaluated under aquatic and terrestrial environment in the Atlantic forest. Wood samples from five species were placed to decompose on the forest floor and twelve of them submerged in a lake, using the litter bags approach to evaluate directly the monthly progressive weight loss. Under lake conditions the decomposition presented tipical a initial period with faster weight loss due to leaching. Correlation analysis showed that wood color is inversely related with decomposition rates of angiosperms $(r=-0,95)$, but wood density is not a good predectivy variable. Decomposition was faster in the aquatic environment, as shown by coefficient $K$ (Olson, 1963) verified for Torresea cearensis 0,63; Cordia goeldiana 0,61; Myroscarpus sp. 0,46; Cedrela sp. 0,35; Swietenia macrophyla 0,31; Araucaria angustifolia 0,28; Pinus sp. 0,26; Phoebe porosa 0,22; Machaerium villosum 0,22; Dalbergia nigra 0,21; Peltogyne discolor 0,20; and Ocotea sp. 0,19. The forest floor values was the following: Torresea cearensis 0,48; Cordia goeldiana 0,43; Phoebe porosa 0,20; Cedrela sp. 0,20; and Pinus 0,16.

Key words: decay, decomposition, wood.

\section{RESUMO}

Foi estudada a cinética de decomposição de laminados de diversas espécies de madeiras em ambientes aquático e terrestre da Mata Atlântica. Amostras de laminados de cinco espécies de madeiras foram expostas sobre o solo da floresta, enquanto amostras de 12 espécies foram estudadas na lagoa presente na mesma área. Foram usadas bolsas de decomposição, "litter bags", para a avaliação direta da perda mensal de peso. No ambiente aquático verificou-se um período típico inicial com maior perda de peso, devido à lixiviação. A análise de correlação mostrou que a cor da madeira é inversamente relacionada à taxa de decomposição das angiospermas $(\mathrm{r}=-0,95)$, mas a densidade das madeiras não é uma variável preditiva adequada. O processo foi mais rápido no ambiente aquático, como demostram os coeficientes de decomposição $K$ (Olson, 1963) obtidos: Torresea cearensis, 0,63; Cordia goeldiana, 0,61; Myroscarpus sp., 0,46; Cedrela sp. 0,35; Swietenia macrophyla 0,31; Araucaria angustifolia 0,28; Pinus sp., 0,26; Phoebe porosa, 0,22; Machaerium villosum, 0,22; Dalbergia nigra, 0,21; Peltogyne discolor, 0,20; e Ocotea sp., 0,19. No solo os valores obtidos são os seguintes: Torresea cearensis, 0,48; Cordia goeldiana, 0,43; Phoebe porosa, 0,20; Cedrela sp., 0,20; e Pinus 0,16.

Palavras-chave: degradação, decomposição, madeira. 


\section{INTRODUÇÃO}

A madeira é um material que apresenta algumas propriedades que a tornaram, desde os primórdios da humanidade, cobiçada e com um lugar de destaque no desenvolvimento da civilização. A resistência mecânica elevada, a facilidade de manuseio, uma resistência química apreciável, um bom isolante elétrico e térmico, a possibilidade de ser encontrada na natureza com ampla variedade e devido à sua ampla faixa de texturas e colorações, na maioria com aromas agradáveis, fizeram da madeira a maior ferramenta usada e desejada pelo Homem.

A superfície do planeta coberta por florestas corresponde a uma área de aproximadamente 4,2 bilhões de hectares, sendo que, de acordo com o Banco Mundial, eram perdidas 15 milhões de ha/ ano de áreas florestadas, até o final da década de 70 (Cockcroft \& Henningsson, 1983).

O Brasil tem uma pequena participação no mercado mundial, de acordo com fontes madeireiras, com 3,5\% distribuídos em $78 \%$ de folhosas e $22 \%$ de coníferas. Não obstante, devido a seu grande potencial madeireiro, o Brasil deverá no futuro próximo ter uma participação mais significativa no abastecimento internacional, principalmente pelo fato de que, em determinados países desenvolvidos, esses recursos estão muito escassos.

Esses valores parecem mostrar que embora a madeira seja um bem renovável, não pode ser encarada como inesgotável. De acordo com Tavares (1984), cerca de $4.000 \mathrm{~m}^{2}$ de florestas tropicais úmidas desapareciam por minuto, tornando-se, assim, imperativo estudos para a resolução do problema com o uso da madeira de forma mais racional, não sendo suficiente apenas os métodos de conservação e reflorestamento, sob risco de surgir uma crise mundial no suprimento desse material.

No Brasil, assim como em muitos países, grandes quantidades de madeiras são submersas em decorrência da criação de reservatórios de água, especialmente com fins de produção de energia elétrica. Nesta situação, grandes quantidades de recursos são perdidos, além do fato de que a decomposição dessa grande quantidade de biomassa pode acarretar problemas para o manejo da qualidade da água do reservatório (Bal et al., 1975; Antonio, 1996). Sabe-se que a decomposição de materiais biológicos depende de uma série de fatores bióticos e abióticos, tais como as condições climáticas, a composição da comunidade decompositora, sua afinidade para com o substrato e as características físicas e químicas do material a ser decomposto (Swift et al., 1979). As madeiras encontram-se entre os materiais biológicos de mais difícil decomposição devido a sua estrutura anatômica e à presenca de grandes quantidades de substâncias recalcitrantes como a lignina, além de outros compostos do metabolismo secundário. Compostos aromáticos acumulados no cerne dos caules e a presença de canais resiníferos em gimnospermas têm a função de conferir resistência a esses orgãos de sustentação, tornando-os, em diferentes graus, imunes às atividades de decompositores (Swift et al., 1979; Minderman, 1968; Delitti, 1995).

O objetivo deste estudo foi investigar a cinética de decomposição de diferentes espécies de madeiras, representando substratos de diversas qualidades. Os estudos foram conduzidos em ambientes terrestre e aquático, representando o primeiro as condições mais usuais em que ocorre a decomposição, em oposição ao ambiente aquático, sítio menos habitual da degradação de madeiras, mas peculiarmente importante, conforme exposto acima.

\section{MATERIAL E MÉTODOS}

\section{Local do Estudo}

A Cidade Universitária Armando Salles de Oliveira, USP, localiza-se a $46^{\circ} 43^{\prime} \mathrm{W}$ e $23^{\circ} 33^{\prime} \mathrm{S}$ e possui uma reserva de 10,2 ha com uma lagoa de $640 \mathrm{~m}^{2}$, na qual foram realizados os experimentos. Trata-se de um relícto de floresta atlântica secundária restrita a uma bacia de drenagem. O clima da região é temperado quente e úmido, sendo nítidos o verão chuvoso e o inverno mais seco (Meguro et al., 1979; Aprile, 1996).

Foram estudados laminados de 12 espécies de madeiras, considerados os mais comercializados pelas empresas madeireiras, sendo elas: Myrocarpus sp. (cabriúva), Cedrela sp. (cedro), Torresea cearensis (cerejeira), Cordia goeldiana (freijó), Phoebe porosa (imbuia), Dalbergia nigra (jacarandá da Bahia), Machaerium villosum (jacarandá paulista), Ocotea sp. (louro preto), Swietenia macrophylia (mogno), Araucaria angustifolia (pinho), Pinus sp. (pinus) e Peltogyne discolor (roxinho). No ambiente terrestre foram estudadas as seguintes 
espécies: Torresea cearensis, Cordia goeldiana, Phoebe porosa, Cedrela sp. e Pinus sp.

\section{Metodologia}

O método escolhido para o trabalho foi o de avaliação direta da perda de massa, com a utilização de bolsas de nylon, "litter bags", de abertura $2 \times 2 \mathrm{~mm}$. Através desse método, um determinado material, cuja massa inicial é conhecida, é colocado dentro das bolsas e exposto à decomposição por períodos de tempo determinados. Os laminados de madeira foram cortados com uma área igual a $50 \mathrm{~cm}^{2}$. Todos foram colocados para secar em temperatura ambiente e depois pesados em uma balança de precisão. Foram realizados ensaios prévios de decomposição e, conhecendo-se a variabilidade desses resultados e a relação entre $\mathrm{N}$ e a variância, pôde-se determinar que nas futuras amostras 10 réplicas seriam suficientes para a obtenção de dados estatisticamente válidos (Sokal \& Rohlf, 1981). Durante um ano foram retiradas mensalmente 10 bolsas de cada espécie de madeira em ambos ambientes, para determinar a progressiva perda de peso.

Os resultados de decomposição foram expressos em porcentagem e foram obtidos a partir da expressão abaixo:

$\%$ Decomp. Acumulada $(\% \mathrm{DA})=100-\frac{\mathrm{Mf} \leftrightarrow 00}{\mathrm{Mi}}$

em que $\mathrm{Mf}=$ massa final e $\mathrm{Mi}=$ massa inicial.

A porcentagem de decomposição acumulada (\%DA), como pode ser visto acima, é o resultado direto da diferença entre massa final e inicial, expressa em porcentagem. Já a porcentagem de decomposição relativa (\%DR) refere-se ao valor obtido para um determinado momento, neste caso para um mês específico, e é obtida da seguinte forma:

$\% \mathrm{DR}_{n}=\% \mathrm{DA}_{n}-\sum_{i=1}^{n-1}(\% \mathrm{DR})_{i}$

Calculamos os coeficientes de decomposição $K$ e $K$ ' segundo Olson (1963). O coeficiente $K$ ' refere-se à quantidade de material decomposto em um dado intervalo de tempo, ao passo que o $K$ se refere à razão instantânea de decomposição, estando ligado ao modelo exponencial.
A relação entre os coeficientes é a seguinte:

$$
\begin{aligned}
& K^{\prime}=\frac{\Delta x}{\mathrm{X} o} \\
& K=-\ln \left(1-K^{\prime}\right)
\end{aligned}
$$

em que $\Delta \mathrm{x}=$ quantidade de material perdido no tempo e $X o=$ quantidade inicial de material.

\section{RESULTADOS E DISCUSSÃO}

As bolsas de decomposição têm sido muito utilizadas para estudos com várias espécies de materiais biológicos, tanto em ecossistemas terrestres como em ecossistemas aquáticos, pois permitem a avaliação direta da perda de massa dos diferentes substratos. No entanto, o fato de não existir uma padronização em relação ao método em si gera uma certa dificuldade em realizar uma comparação entre a velocidade de decomposição das várias espécies estudadas e entre os diversos ambientes. Em relação ao tamanho da malha usada na confecção dos sacos foi observado que, se as aberturas das malhas forem muito finas $(<0,3 \mathrm{~mm})$, poderiam vir a excluir a entrada da macrofauna, enquanto que, se as aberturas das malhas forem muito grossas $(>3,0 \mathrm{~mm})$, permitiriam a entrada de grande parte dos invertebrados e de alguns vertebrados, como peixes pequenos, que podem se tornar uma visita indesejável às bolsas, pois são capazes de destruir parcial ou totalmente o material de pesquisa (Swift et al., 1979).

A decomposição normalmente não é contínua. O curso de seu tempo deve ser representado como uma sucessão de fases em plena atividade, com intervalos de inibição, devido a limitação ou inibição completa de processos físicos, químicos ou biológicos no processo de decomposição. As Tabelas 1 e 2 mostram a porcentagem média de decomposição acumulada (\%XDA) e a porcentagem de decomposição relativa (\%DR), obtidas a partir das Equações 1 e 2, respectivamente, além dos parâmetros estatísticos de tendência central e de dispersão. Os resultados são referentes ao último mês de coleta. No geral, os desvios $(S)$ foram baixos, sendo baixos também os coeficientes de variação $(\mathrm{CV})$. A Tabela 3 mostra os coeficientes de decomposição $K$ 'e $K$ obtidos a partir das Equações 3 e 4 para o décima segundo mês de coleta. 
TABELA 1

Resultados do estudo de decomposição dos laminados de madeira em ambiente terrestre, para o décimo segundo mês de coleta, com suas respectivas análises estatísticas.

\begin{tabular}{|l|l|l|l|l|l|l|l|l|}
\hline Laminado & $\mathbf{N}$ & \%X DA & \%DR & $\boldsymbol{S}$ & $\boldsymbol{C V}$ & $\boldsymbol{S} \boldsymbol{C}$ & Mín. & Máx. \\
\hline Cedro & 10 & 18,341 & 0,572 & 2,380 & 12,98 & 0,752 & 13,30 & 23,40 \\
\hline Cerejeira & 10 & 38,021 & 0,466 & 1,496 & 3,93 & 0,473 & 35,00 & 41,03 \\
\hline Freijó & 10 & 34,979 & 1,757 & 2,225 & 6,36 & 0,703 & 30,67 & 39,86 \\
\hline Imbuia & 10 & 18,193 & 1,176 & 3,164 & 17,39 & 1,000 & 12,20 & 24,21 \\
\hline Pinus & 10 & 14,921 & 1,143 & 2,414 & 16,18 & 0,763 & 10,90 & 18,95 \\
\hline
\end{tabular}

TABELA 2

Resultados do estudo de decomposição dos laminados de madeira em ambiente aquático, para o décimo segundo mês de coleta, com suas respectivas análises estatísticas.

\begin{tabular}{|c|c|c|c|c|c|c|c|c|}
\hline Laminado & $\mathbf{N}$ & $\% \mathbf{D A}$ & $\% \mathrm{DR}$ & $S$ & $C V$ & $S x$ & Mín. & Máx. \\
\hline Cabreúva & 9 & 36,923 & 7,535 & 2,237 & 6,06 & 0,745 & 32,87 & 40,60 \\
\hline Cedro & 10 & 29,659 & 6,673 & 1,685 & 5,68 & 0,533 & 25,99 & 32,32 \\
\hline Cerejeira & 10 & 46,570 & 7,341 & 2,862 & 6,14 & 0,905 & 40,50 & 52,64 \\
\hline Freijó & 10 & 45,795 & 8,244 & 2,867 & 6,26 & 0,907 & 40,39 & 51,69 \\
\hline Imbuia & 10 & 19,879 & 0,640 & 2,654 & 13,35 & 0,839 & 15,80 & 23,96 \\
\hline Jac. Bahia & 10 & 18,820 & 4,050 & 1,704 & 9,05 & 0,538 & 15,81 & 22,83 \\
\hline Jac. paulista & 10 & 19,808 & 2,876 & 2,353 & 11,88 & 0,744 & 15,49 & 24,82 \\
\hline Louro-preto & 10 & 17,411 & 4,143 & 1,963 & 11,27 & 0,620 & 13,37 & 21,45 \\
\hline Mogno & 10 & 27,240 & 4,328 & 0,097 & 0,35 & 0,031 & 27,04 & 27,44 \\
\hline Pinho & 10 & 24,240 & 4,131 & 3,314 & 13,67 & 1,047 & 20,12 & 28,39 \\
\hline Pinus & 10 & 22,921 & 3,692 & 1,341 & 5,85 & 0,424 & 20,90 & 24,95 \\
\hline Rouxinho & 10 & 17,881 & 2,239 & 3,295 & 18,43 & 1,042 & 10,89 & 24,87 \\
\hline
\end{tabular}

$\mathrm{N}=$ Tamanho da amostra; \%XDA = Porcentagem média de decomposição acumulada; \%DR = porcentagem de decomposição relativa; $S=$ Desvio padrão; $C V=$ Coeficiente de variação; $S x=$ Erro padrão da média; Mín. = Valor mínimo encontrado; e Máx. = Valor máximo encontrado.

A dinâmica da água e o regime térmico em que a amostra está submetida controlam a taxa de desaparecimento da matéria orgânica e da liberação dos elementos que a constituem. Entretanto, apesar de o inverno em São Paulo ser frio e seco, e nestas condições ser possível uma diminuição da transformação da matéria orgânica pelo menor fluxo de água, associada à menor atividade metabólica dos organismos decompositores, a variação sazonal não foi muito perceptível nos estudos no ambiente aquático.

Acredita-se que a palatabilidade, associada à densidade do material, tenham sido fatores que contribuíram para que as madeiras apresentassem resultados bastante distintos, tanto em ambiente aquático como no terrestre.

A palatabilidade pode ser um dos fatores que determinam uma maior preferência dos decompositores por um determinado tipo de laminado. A qualidade do material em decomposição é muito importante, pois seu conteúdo de energia e nutrientes pode determinar o grau de palatabilidade aos detritívoros. É amplamente conhecido que a degradação da lignina e dos compostos fenólicos que produzem taninos diminuem a palatabilidade. Heath \& King (1964) em seus estudos com polifenóis comprovam esse fato. De acordo ainda com os autores, concentrações de ácidos gálico e protocatecóico 
também têm essa propriedade, tornando o material menos sujeito ao ataque de invertebrados. Isso explicaria os resultados obtidos nas Figs. 1 e 2, em que os laminados de cores mais escuras, nos quais tais compostos estão em maior quantidade, foram os mais resistentes. Esses compostos dão uma maior dureza e resistência às madeiras, tornando-as mais refratárias aos processos de lixiviação, além de menos palatável aos organismos decompositores e menos acessível aos sistemas enzimáticos de digestão (Swift et al., 1979).

Acredita-se que a densidade seja uma importante característica determinante da velocidade de decomposição de um determinado material (Dickinson, 1974). Sabendo disso, procurou-se criar uma classificação das madeiras estudadas, de acordo com a densidade e a coloração. Para a classificação por índices de coloração dos laminados estudados utilizou-se a escala de Munsell (1975); e as densidades das madeiras foram as citadas por Rizzini (1990). A tonalidade de um laminado está associada à concentração de compostos do metabolismo secundário, como polifenóis, taninos etc., que têm grande influência na palatabilidade e conseqüentemente na cinética de decomposição (Heath \& King, 1964).

Testes de correlação (r) foram feitos com o objetivo de certificar-se da existência de uma associação entre as taxas de decomposição, densidade e coloração do material (Figs. 3, 4 e 5). Pode-se verificar que o $r$ determinado para os dois ambientes foi negativo, com exceção do valor determinado para a correlação entre o $K \infty$ Densidade, no solo. Esse fato indica um comportamento inversamente proporcional entre tais parâmetros. Nota-se que, de um modo geral, as madeiras mais claras e menos densas tiveram realmente maiores taxas de decomposição, com exceção do pinus e pinho. No entanto, os valores calculados para $\mathrm{r}$ são relativamente baixos, e a análise dos valores de $\mathrm{p}$ indica uma forte tendência a crer que os eventos podem ser considerados independentes.

\section{TABELA 3}

Valores de $K$ e $K$ ' para as madeiras estudadas nos ambientes aquático e terrestre, referentes ao décimo segundo mês de coleta.

\begin{tabular}{|c|c|c|c|}
\hline Laminado & $\mathbf{N}$ & $\boldsymbol{K}$ & $K^{\prime}$ \\
\hline \multicolumn{4}{|c|}{ Ambiente aquático } \\
\hline Cabreúva & 9 & 0,460 & 0,369 \\
\hline Cedro & 10 & 0,351 & 0,296 \\
\hline Cerejeira & 10 & 0,627 & 0,466 \\
\hline Freijó & 10 & 0,612 & 0,458 \\
\hline Imbuia & 10 & 0,220 & 0,198 \\
\hline Jac. Bahia & 10 & 0,208 & 0,188 \\
\hline Jac. paulista & 10 & 0,220 & 0,198 \\
\hline Louro-preto & 10 & 0,191 & 0,174 \\
\hline Mogno & 10 & 0,317 & 0,272 \\
\hline Pinho & 10 & 0,277 & 0,242 \\
\hline Pinus & 10 & 0,260 & 0,229 \\
\hline Rouxinho & 10 & 0,197 & 0,179 \\
\hline \multicolumn{4}{|c|}{ Ambiente terrestre } \\
\hline Cedro & 10 & 0,202 & 0,183 \\
\hline Cerejeira & 10 & 0,478 & 0,380 \\
\hline Freijó & 10 & 0,429 & 0,349 \\
\hline Imbuia & 10 & 0,201 & 0,182 \\
\hline Pinus & 10 & 0,161 & 0,149 \\
\hline
\end{tabular}




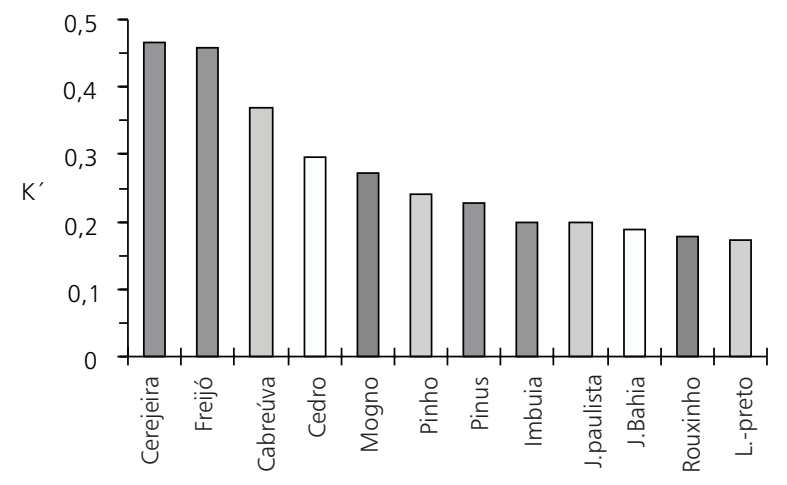

Fig. 1 - Gráfico mostrando o coeficiente de decomposição $K^{\prime}$, referente ao décimo segundo mês, para os laminados em ambiente aquático.

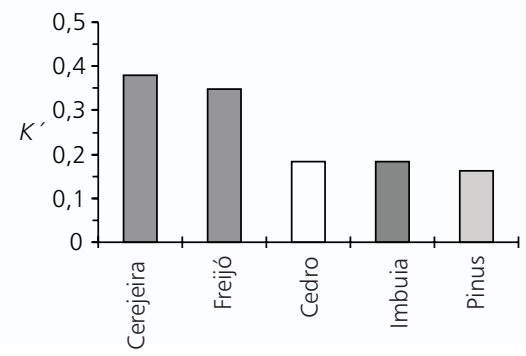

Fig. 2 - Gráfico mostrando o coeficiente de decomposição $K^{\prime}$, referente ao décimo segundo mês, para os laminados em ambiente terrestre.
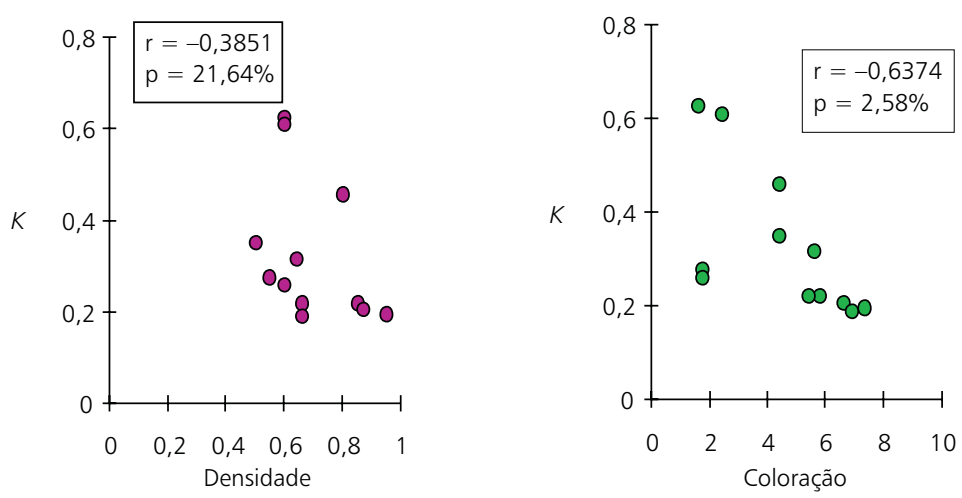

Fig. 3 - Correlação entre a razão instantânea de decomposição $(K)$, a densidade e a coloração dos laminados de madeira em ambiente aquático. 

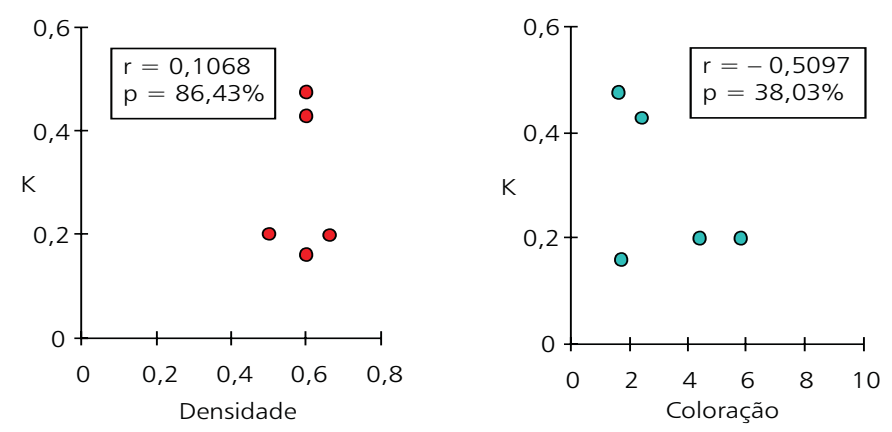

Fig. 4 - Correlação entre a razão instantânea de decomposição $(K)$, a densidade e a coloração dos laminados de madeira em ambiente terrestre.
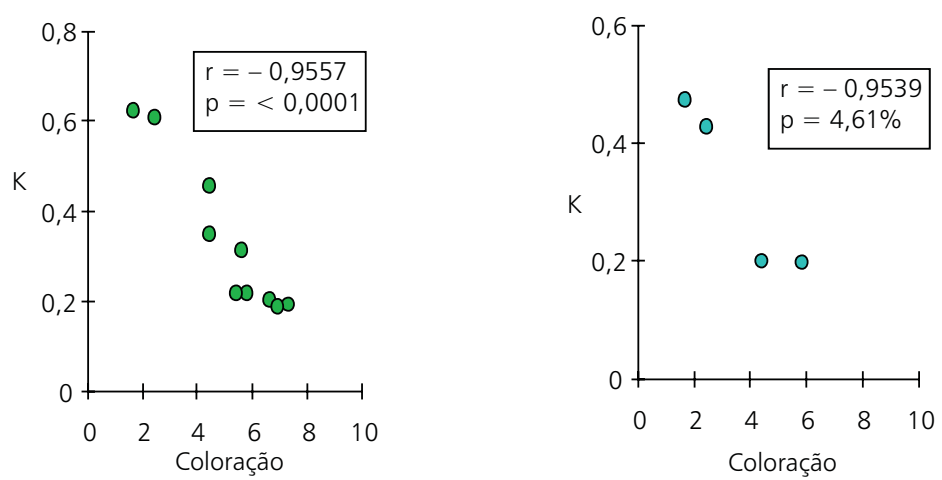

Fig. 5 - Correlação entre a razão instantânea de decomposição $(K)$ e a coloração dos laminados de madeira nos ambientes aquático e terrestre sem as espécies pinus e pinho.

Talvez esse comportamento tenha sido influenciado pelos resultados do pinho e do pinus, que foram exceções ao padrão de decomposição. Para esclarecer esse ponto foram retiradas as duas espécies da curva de correlação. O resultado foi um aumento significativo do r, entre $K$ e a coloração, de $-0,5097$ para $-0,9539$, e um decréscimo do $p$ de 38 para 4,6\% (Fig. 5). Isso vem demonstrar que realmente existe uma tendência a que os eventos sejam inversamente dependentes, porém, quando anexamos na análise os valores do pinho e do pinus há um deslocamento da curva de correlação, diminuindo o grau de associação.

O mesmo comportamento foi observado no ambiente terrestre, em que o $r$ também sofreu um aumento de $-0,5097$ para $-0,9539$ com a retirada dos resultados do pinus (Fig. 5). A validade dessa análise deve-se ao fato dessas duas madeiras pertencerem a um grupo taxonômico muito distinto (Gymnospermae), que apresenta constituição anatômica particular e compostos quími- cos típicos, cuja coloração não é destacada, apesar de conferirem resistência à decomposição. Além disso, as coníferas têm maior concentração de lignina que as angiospermas, sendo esta uma das substâncias de mais lenta decomposição (Minderman, 1968; Martitegui et al., 1994).

A correlação entre $K$ e a densidade do laminado não foi satisfatória, apresentando resultados baixos para os dois ambientes de estudo. Outra análise de correlação baseada em dados mais precisos sobre a densidade desses laminados poderá fornecer resultados distintos, já que até o momento foram usados apenas os dados de bibliografia (Rizzini, 1990).

\section{CONCLUSÕES}

A cinética de decomposição dos laminados de madeira foi maior no ambiente aquático que no ambiente terrestre, em decorrência principalmente dos processos de lixiviação. Os laminados 
de coloração clara e densidade baixa foram, em geral, os que apresentaram maiores velocidade de decomposição. A cerejeira e o freijó foram as espécies de laminados que apresentaram os maiores valores de decomposição durante os 12 meses de estudos, nos dois ecossistemas. Os laminados mais resistentes ao processo de decomposição foram: para o ambiente terrestre, a imbuia e o pinus; para o ambiente aquático, o rouxinho e o louro-preto. A análise de correlação entre $K$ e a coloração do laminado mostrou que, quando todos os laminados são analisados conjuntamente, os resultados de $r$ são baixos, indicando uma correlação bastante fraca tanto para o ambiente aquático como para o terrestre. Quando a análise se restringe às angiospermas, os resultados mostram-se satisfatórios, com alto coeficiente de correlação em ambos ambientes. A análise de correlação entre $K$ e a densidade do laminado apresentou resultados de $\mathrm{r}$ baixos, para ambos os ambientes, indicando não haver correlação entre a taxa de decomposição de um laminado e a sua densidade específica.

Agradecimentos - A CAPES pela bolsa de estudo concedida; a prof. Dra. V. A. Alfonso e pesq. H. L. Oliveira Filho (Depto. de Botânica - USP) pela identificação do material; à bióloga L. A. Silva.

\section{REFERÊNCIAS BIBLIOGRÁFICAS}

ANTONIO, R. M., 1996, Estimativa da Capacidade Heterotrófica do Lago Infernão (Estação Ecológica de Jataí, SP). Dissertação de Mestrado. PPGERN, UFSCar, São Carlos.

APRILE, F. M., 1996, Cinética de decomposição de madeiras em ambiente aquático e terrestre. Dissertação de Mestrado. Departamento de Ecologia Geral, Instituto de Biociências, USP, São Paulo.

BAL, J., WELDON, C. \& CROCKER, B., 1975, Effectes of original vegetation on reservoir water quality. Tech. Rep. Tex. A \& M Water Resour. Inst., 64.

COCKCROFT, R. \& HENNINGSSON, B. O., 1983, The global needs of wood preservation. The Int.. J. Wood Preserv., Guernsey, 3(2): 65-71.

DELITTI, W. B. C., 1995, Estudos de ciclagem de nutrientes: Instrumentos para análise funcional de ecossistemas terrestres. In: F. A. Esteves (ed.), Estrutura, Funcionamento e Manejo de Ecossistemas. UFRJ, Ecologia Brasiliensis, 1: 469-486.

DICKINSON, C. H., 1974, Decomposition of litter in soil. Biology of plnat litter decomposition. C. H. Dickinson e G. J. F. Pugh (eds.), vol. 2, p. 633-658. London, Academic Press.
HEATH, G. W. \& KING, H. G. C., 1964, Litter breakdown in deciduous forest soils. Int. Congr. Soil Sci., 8(3): 979987.

MARTITEGUi , A., ALVAREZ, M. A. G., GALlEGO, G. M. \& GUTTIEREZ, J. O., 1994, Guia de la madera. AITIM, Madrid, pp. 21-72.

MEGURO, M., VINUEZA, G. N. \& DELITTI, W. B. C., 1979, Ciclagem de Nutrientes Minerais na Mata Mesófila Secundária. I - Produçåo e Conteúdo de Nutrientes Minerais do Folhedo. Bolm Botânica, USP, 7: 13-31.

MINDERMANN, G., 1968, Addition, decomposition and accumulation of organic matter in forests. J. Ecol., 56: 355-562.

MUNSELL' SOIL COLOR CHARTS, 1975, Macbeth a division of Kollmorgen Corporation.

OLSON, J. S., 1963, Energy storage and the balance of producers and decomposers in ecological systems. Ecology, 44(2): 322-332.

RIZZINI, C. T., 1990, Árvores e Madeiras úteis do Brasil. Manual de dendrologia brasileira, 2a ed., São Paulo, Edgard Blücher, 304p.

SOKAL, R. R. \& ROHLF, F. J., 1981, Biometry. W. H. Freeman Company, New York, 859p.

SWIFT, M. J., HEAL, D. W. \& ANDERSON, J. M., 1979 , Studies in Ecology-Decomposition in Terrestrial and Aquatic Ecosystems. Oxford, Blackwell.

TAVARES, O. D. P., 1984, O incentivo ao reflorestamento no Brasil. In: II Seminário sobre exportação de madeiras tropicais. Resumos, São Luiz, Maranhão, 16-17 de outubro, $7 \mathrm{p}$. 